\title{
Acúmulo de forragem e características do solo e da planta no estabelecimento de capim-massai com diferentes níveis de saturação por bases, fósforo e nitrogênio
}

\author{
Edimilson Volpe ${ }^{1}$, Marlene Estevão Marchetti ${ }^{2}$, Manuel Cláudio Motta Macedo ${ }^{3}$, Beatriz Lempp ${ }^{2}$ \\ 1 Agência de Desenvolvimento Agrário e Extensão Rural de Mato Grosso do Sul. \\ 2 Universidade Federal da Grande Dourados - UFGD. \\ ${ }^{3}$ Embrapa Gado de Corte.
}

RESUMO - Foi conduzido um experimento em Latossolo de Cerrado visando testar a combinação de níveis de saturação por bases $(20,40,60$ e $80 \%)$, doses de fósforo $\left(0,80,160\right.$ e $\left.240 \mathrm{~kg} / \mathrm{ha} \mathrm{de} \mathrm{P}_{2} \mathrm{O}_{5}\right)$ e nitrogênio $(0,100,200$ e $300 \mathrm{~kg} / \mathrm{ha}) \mathrm{no}$ estabelecimento de capim-massai. Foram avaliados o acúmulo de massa seca verde (MSV) da forrageira, as características químicas do solo, as concentrações foliares de macronutrientes, os teores de proteína bruta (PB) e a digestibilidade in vitro da matéria orgânica (DIVMO). Utilizou-se o delineamento de blocos casualizados no esquema fatorial fracionário $(1 / 2) 4^{3}$, com duas repetições e quatro blocos. Foram realizados dois cortes em aproximadamente 200 dias. O acúmulo de MSV respondeu principalmente ao fósforo $(\mathrm{P}) \mathrm{e}$, em segundo lugar, à saturação por bases. Não houve resposta significativa ao nitrogênio $(\mathrm{N})$. A dose de $\mathrm{P}_{2} \mathrm{O}_{5}$ para o máximo acúmulo de MSV foi de $237 \mathrm{~kg} / \mathrm{ha}$ e a dose econômica, de $185 \mathrm{~kg} / \mathrm{ha}$, com saturação por bases no solo de $39 \%$. A fertilidade do solo aumentou com o acréscimo de saturação por bases e P. As concentrações foliares de $\mathrm{P}$, Ca e Mg elevaram-se com o aumento dos níveis de saturação por bases e P e a concentração de N aumentou com a adubação nitrogenada e com o incremento da saturação por bases. A fração folha correspondeu a 77,50\% da MSV e apresentou valores adequados de DIVMO e PB, que aumentaram significativamente com o aumento da adubação nitrogenada e da saturação por bases.

Palavras-chave: folhas diagnósticas, latossolo, massa seca verde, Panicum maximum

\section{Forage accumulation and plant and soil characteristics according to soil base saturation, rates of phosphorus and nitrogen in the establishment of massaigrass}

\begin{abstract}
It was carried out an experiment to test four levels of soil base saturation (20,40,60, and 80\%), four rates of phosphorus $\left(0,80,160\right.$, and $240 \mathrm{~kg} / \mathrm{ha}$ of $\left.\mathrm{P}_{2} \mathrm{O}_{5}\right)$ and four rates of nitrogen $(0,100,200$ and $300 \mathrm{~kg} / \mathrm{ha})$ during the phase of establishment of Panicum maximumcv Massai, in an Oxisol of Brazilian Cerrado. The experimental design was a randomized uncompleted block, in fractionated factorial type $(1 / 2) 4^{3}$, with four blocks and two replications. Two cuts along 200 days were performed. The evaluated aspects were green dry matter yield (GDM), soil fertility changes, concentration of macronutrients in diagnostic leaves, crude protein $(\mathrm{CP})$ concentration and in vitro organic matter digestibility (IVOMD). It was verified that GDM yield responded mainly to phosphorus (P) fertilization and, in second place, to soil base saturation (V). The nitrogen $(\mathrm{N})$ did not present significant effect in GDM yield. The P rate for highest physical yield was $237 \mathrm{~kg} / \mathrm{ha}$ and the economical rate was $185 \mathrm{~kg} / \mathrm{ha}$, both in $39 \%$ of V in the soil. Soil fertility improved with the V and P increment. The concentration of macronutrients in diagnostic leaves increased with the increment of $\mathrm{V}, \mathrm{P}$, and $\mathrm{N}$. It was verified high percentage of leaves in GDM and suitable IVOMD and CP concentration in the GDM of leaves. IVOMD and CP increased significantly with $\mathrm{N}$ fertilization and increment of $\mathrm{V}$.
\end{abstract}

Key Words: diagnostic leaves, green dry matter, Oxisol, Panicum maximum

\section{Introdução}

Nos solos de Cerrado, predominam boas propriedades físicas e baixa fertilidade natural. As pastagens cultivadas nesse bioma são constituídas principalmente de gramíneas do gênero Brachiaria em razão de sua adap- tação ao ambiente. As práticas de correção e adubação são pouco utilizadas, caracterizando um sistema de exploração extrativista, principal fator responsável pela degradação destas pastagens.

A correção e adubação do solo são consideradas práticas prioritárias nos estudos de formação e renovação e/ou

Este artigo foi recebido em 14/11/2006 e aprovado em 13/8/2007.

Correspondências devem ser enviadas para edvolpe@terra.com.br. 
recuperação de pastagens (Luz et al., 2000). A calagem é a primeira ação a ser implementada, mas necessita de maiores estudos, pois as respostas a esta prática têm sido diferenciadas (Cruz et al., 1994; Oliveira et al., 2003; Paulino et al., 2006), provavelmente em virtude de diferenças de solo e da variabilidade de tolerância à acidez do solo das principais gramíneas forrageiras tropicais. Macedo (2005) considera que a controvérsia reside, no Cerrado, em grande parte na natureza da fração argila dos Latossolos, cuja reação à calagem é diferenciada de solos de outras regiões, além da alta tolerância à acidez das forrageiras mais utilizadas neste ambiente.

O fósforo (P) é o nutriente mais limitante no estabelecimento de pastagens no Cerrado, em virtude dos baixos teores e da alta capacidade de fixação dos solos. O P também é importante na formação do sistema radicular, uma vez que sua absorção é limitada pela baixa mobilidade no solo e pelo reduzido sistema radicular inicial das plantas; níveis críticos de $\mathrm{P}$ no solo diminuem exponencialmente com o avanço da idade das plantas (Santos et al., 2002).

No estabelecimento da pastagem, o nitrogênio $(\mathrm{N})$ é considerado importante em sistemas mais intensivos e/ou em solos com baixo teor de matéria orgânica, quando normalmente se recomenda a adubação nitrogenada em cobertura. No entanto, podem ocorrer grandes perdas deste nutriente, especialmente por volatilização, além de grandes variações das respostas em produtividade das gramíneas forrageiras, tratando-se dos aspectos mais críticos para a utilização de $\mathrm{N}$ (Martha Jr. et al., 2004a,b). Mesmo em sistemas intensivos, tem se verificado falta de resposta ao $\mathrm{N}$ no estabelecimento de capins (Saraiva \& Carvalho, 1991).

$\mathrm{Na}$ adubação de pastagens deve-se considerar a restrição econômica; o critério matemático mais tradicional fundamenta-se na lei dos rendimentos decrescentes (Van Raij, 1991). No limite, quando o valor do aumento da produção é igual ao custo do fertilizante, atinge-se a chamada dose econômica pela análise marginal (Noronha, 1984). Esta análise tem sido utilizada para forrageiras e culturas anuais em alguns trabalhos (Primavesi et al., 2004).

Em 2001 foi lançada uma nova forrageira pertencente à espécie Panicum maximum: o cultivar Massai, um híbrido natural entre $P$. maximum e Panicum infestum (Embrapa, 2001) que parece constituir-se em opção importante para a diversificação das pastagens no Cerrado. Em relação aos outros cultivares de Panicum esse capim apre senta diferenças morfológicas acentuadas, maior tolerância à acidez, a reduzida fertilidade dos solos e a outros estresses ambientais, mas valor nutritivo inferior(Valentim et al., 2001; Brâncio et al., 2003).
Objetivou-se neste trabalho avaliar o acúmulo de forragem e as características da planta (concentração de macronutrientes, teores de proteína bruta, digestibilidade nas folhas e composição morfológica da forrageira) e do solo (pH, saturação por bases, teores de fósforo, cálcio e magnésio), bem como as máximas doses agronômicas e econômicas, na fase de estabelecimento do capim-massai cultivado em diferentes níveis de saturação por bases (V) e doses de $\mathrm{P}$ e $\mathrm{N}$ em Latossolo típico do Cerrado.

\section{Material e Métodos}

O experimento foi realizado em Latossolo Vermelho Distrófico da classe textural argila arenosa, na Embrapa Gado de Corte, em Campo Grande, MS (530 m de altitude; $\left.20^{\circ} 27^{\prime} \mathrm{S} ; 5^{\circ} 37^{\prime \prime} \mathrm{W}\right)$, no período de setembro de 2003 a julho de 2004. O clima local é o tropical chuvoso de Cerrado, com déficit hídrico no período de outono-inverno, temperatura média de $23^{\circ} \mathrm{C}$ e precipitação média anual (período: 19732003) de $1.528 \mathrm{~mm}$ (Figura 1).

Foram testados quatro níveis de saturação por bases (20, 40, 60 e $80 \%)$, quatro doses de $\mathrm{P}(0,80,160$ e $240 \mathrm{~kg} / \mathrm{ha}$ de $\left.\mathrm{P}_{2} \mathrm{O}_{5}\right)$ e quatro doses de $\mathrm{N}(0,100,200$ e $300 \mathrm{~kg} / \mathrm{ha})$ utilizando como fontes, respectivamente, calcário dolomítico com PRNT de $80 \%$, superfosfato triplo e uréia. O ensaio foi implantado em área experimental onde, anteriormente, foram testados quatro níveis de saturação por bases (V) para diversas forrageiras, com exceção do bloco 4, que foi implantado dentro da área experimental em solo coberto por plantas infestantes (Brachiaria). Desta forma, nos blocos 1 a 3, utilizaram-se parcelas de tamanho igual ao do experimento anterior e os níveis crescentes de saturação por bases do experimento incorporaram os níveis crescentes do experimento anterior, de forma a não ocorrer efeito residual.

O delineamento experimental utilizado foi em blocos ao acaso, no esquema fatorial fracionário $(1 / 2) 4^{3}$ (Andrade \& Noleto, 1986), em um total de 32 parcelas por repetição (Tabela 1). Foram utilizadas duas repetições, totalizando quatro blocos com 64 parcelas. As parcelas mediram $4 \times 6 \mathrm{~m}$. Foram retiradas amostras de solo nas 64 parcelas do experimento para análise química e duas amostras em toda a área do experimento para análise textural, na profundidade de 0 a $20 \mathrm{~cm}$, em julho de 2003 .

Os resultados das análises do solo (Tabela 2) foram obtidos conforme descrito por Embrapa (1997). A dose de calcário a ser utilizada foi obtida por incubação, em laboratório, do solo amostrado com o calcário utilizado no experimento. A calagem destinada a atingir os níveis préestabelecidos de saturação por bases foi realizada em setembro de 2003, em cada parcela. O calcário aplicado foi 
incorporado com enxada rotativa até a profundidade aproximada de $20 \mathrm{~cm}$.

As operações de adubação e plantio foram realizadas de 15 a 19 de dezembro de 2003. Todas as parcelas receberam aplicação de $100 \mathrm{~kg} /$ ha de $\mathrm{K}_{2} \mathrm{O}$ na forma de cloreto de potássio $(\mathrm{KCl}), 100 \mathrm{~kg} / \mathrm{ha}$ de enxofre elementar e micronutrientes: 0,$2 ; 2,0 ; 2,0$ e $1,0 \mathrm{~kg} / \mathrm{ha}$, respectivamente, de molibdênio (molibdato de sódio), zinco e cobre (sulfatos) e boro (bórax). A adubação fosfatada também foi realizada nessa ocasião.

Os fertilizantes foram aplicados a lanço e incorporados com enxada rotativa $(0-20 \mathrm{~cm})$. O capim-massai foi semeado em linhas espaçadas de $20 \mathrm{~cm}$ utilizando-se $3 \mathrm{~kg} / \mathrm{ha}$ de sementes puras viáveis, em sulcos com 2-3 cm de profundidade. A aplicação de $\mathrm{N}$ foi feita parte no plantio $(20 \mathrm{~kg} / \mathrm{ha})$, exceto na dose 0 de $\mathrm{N}$, juntamente com os outros fertilizantes. O restante de $\mathrm{N}$ foi utilizado em cobertura, de modo que cada dose foi dividida em duas aplicações de quantidades iguais, aplicadas em 10/2/2004 e 10/4/2004.

As amostragens de acúmulo de biomassa foram realizadas em 2/4/2004 (Período de 26/12/2003 - emergência média - a 2/4/2004 = 96 dias) e 23/7/2004 (período de 10/4/ 2004 - dia da uniformização - a 23/7/2004 = 104 dias). A amostragem foi feita retirando-se duas amostras de forragem a $15 \mathrm{~cm}$ do solo em cada parcela, medindo $1 \mathrm{~m}^{2}$ cada amostra, que foram pesadas verdes e uma delas submetida à secagem a $65^{\circ} \mathrm{C}$ durante 72 horas e pesada para determinação da umidade e cálculo da produção de massa seca. Uma subamostra de aproximadamente $500 \mathrm{~g}$ foi retirada de cada amostra não submetida à secagem para a separação morfológica do capim (folhas, colmos + bainhas e material senescente). Após a separação, foram submetidas à secagem e pesadas, de forma idêntica às amostras completas. As
Tabela 1 - Descrição dos tratamentos nos dois blocos de cada repetição com as saturações por bases-alvos e as quantidades totais dos nutrientes aplicados

\begin{tabular}{|c|c|c|}
\hline \multirow[t]{2}{*}{ Tratamento } & Saturação por bases & Nutriente \\
\hline & $\%$ & $\mathrm{P}_{2} \mathrm{O}_{5}$ \\
\hline
\end{tabular}

Blocos 1/3

\begin{tabular}{rrrr}
000 & 20 & 0 & 0 \\
011 & 20 & 80 & 100 \\
022 & 20 & 160 & 200 \\
033 & 20 & 240 & 300 \\
101 & 40 & 0 & 100 \\
110 & 40 & 80 & 0 \\
123 & 40 & 160 & 300 \\
132 & 40 & 240 & 200 \\
202 & 60 & 0 & 200 \\
213 & 60 & 80 & 300 \\
220 & 60 & 160 & 0 \\
231 & 60 & 240 & 100 \\
303 & 80 & 0 & 300 \\
312 & 80 & 80 & 200 \\
321 & 80 & 160 & 100 \\
330 & 80 & 240 & 0 \\
\hline
\end{tabular}

Blocos 2/4

\begin{tabular}{rrrr}
003 & 20 & 0 & 300 \\
012 & 20 & 80 & 200 \\
021 & 20 & 160 & 100 \\
030 & 20 & 240 & 0 \\
102 & 40 & 0 & 200 \\
120 & 40 & 160 & 0 \\
113 & 40 & 80 & 300 \\
131 & 40 & 240 & 100 \\
201 & 60 & 0 & 100 \\
210 & 60 & 80 & 0 \\
223 & 60 & 160 & 300 \\
232 & 60 & 240 & 200 \\
300 & 80 & 0 & 0 \\
311 & 80 & 80 & 100 \\
322 & 80 & 160 & 200 \\
333 & 80 & 240 & 300 \\
\hline
\end{tabular}

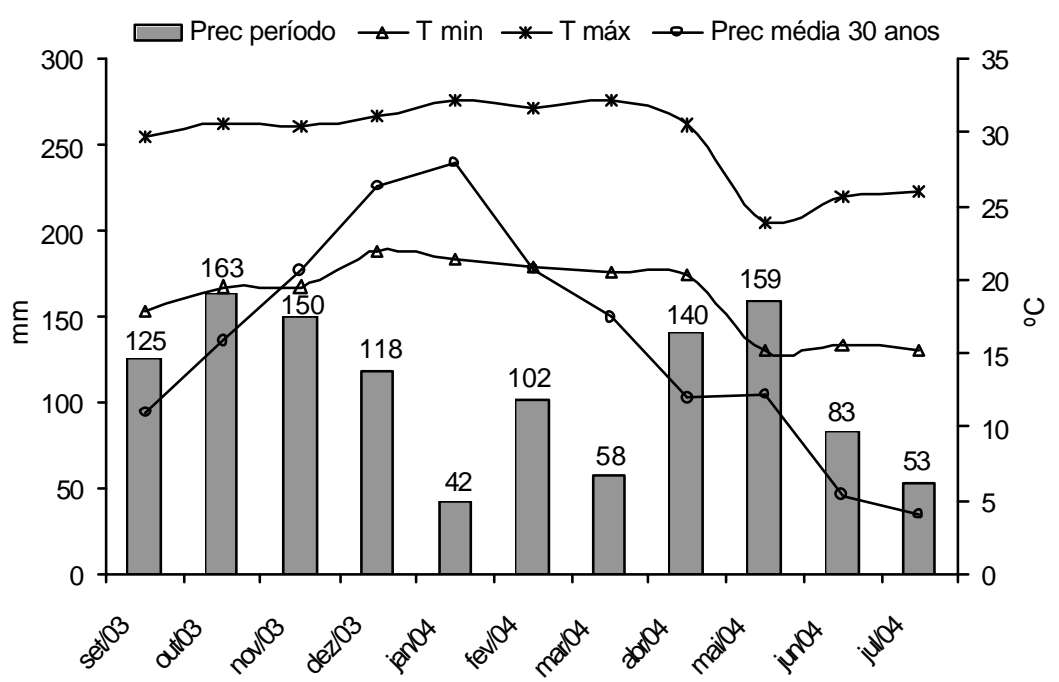

Figura 1 - Precipitação (Prec) pluvial $(\mathrm{mm})$ e temperaturas médias máximas e mínimas mensais $\left({ }^{\circ} \mathrm{C}\right)$ durante o experimento e média de precipitação de 30 anos (1973 a 2003). 
Tabela 2 - Resultados das análises químicas e textural do solo na profundidade $0-20 \mathrm{~cm}$, pré-instalação do experimento

\begin{tabular}{|c|c|c|c|c|c|c|c|c|c|c|}
\hline \multicolumn{4}{|c|}{ Calagem anterior } & \multicolumn{7}{|c|}{ Característica química } \\
\hline & \multirow[t]{2}{*}{$\mathrm{pH} \mathrm{CaCl}{ }_{2}$} & \multirow[t]{2}{*}{$\mathrm{Ca}$} & $\mathrm{Mg}$ & $\mathrm{Al}$ & $\mathrm{H}+\mathrm{Al}$ & $\mathrm{T}$ & \multirow[t]{2}{*}{$\mathrm{K}$} & \multirow{2}{*}{$\begin{array}{l}\mathrm{V} \\
\%\end{array}$} & \multirow{2}{*}{\multicolumn{2}{|c|}{$-\mathrm{mg} / \mathrm{dm}^{3}-$}} \\
\hline & & & & - - - & $/ \mathrm{dm}^{3}-$ & & & & & \\
\hline Não ${ }^{1}$ & 4,37 & 6,2 & 5,1 & 4,4 & 62,8 & 75,5 & 1,5 & 17 & 2,06 & 35,9 \\
\hline $\mathrm{CV}(\%)$ & 3 & 35 & 35 & 31 & 14 & 10 & 24 & 28 & 35 & 8 \\
\hline $\operatorname{Sim}^{2}$ & 4,86 & 13,4 & 9,3 & 1,7 & 46,6 & 71,0 & 1,7 & 34 & 2,76 & 34,4 \\
\hline \multirow[t]{4}{*}{$\mathrm{CV}(\%)$} & 7 & 36 & 39 & 75 & 28 & 8 & 30 & 33 & 28 & 7 \\
\hline & & \multicolumn{9}{|c|}{ Textura $^{3}$} \\
\hline & & \multicolumn{3}{|l|}{ Areia } & \multicolumn{2}{|c|}{$\begin{array}{l}\text { Silte } \\
-\mathrm{g} / \mathrm{kg}\end{array}$} & & & \multicolumn{2}{|c|}{ Argila } \\
\hline & & \multicolumn{2}{|l|}{567} & & \multicolumn{2}{|c|}{62} & & & \multicolumn{2}{|c|}{371} \\
\hline
\end{tabular}

${ }^{1}$ Médias de 36 parcelas que não haviam recebido calagem anteriormente.

${ }^{2}$ Médias de 28 parcelas que receberam calcário anteriormente.

${ }^{3}$ Médias de duas repetições.

folhas secas desta subamostra foram destinadas às análises de proteína bruta (PB) e digestibilidade in vitro da matéria orgânica (DIVMO) utilizando-se o sistema de espectroscopia de Reflectância no Infravermelho Proximal (NIRS), de acordo com os procedimentos de Marten et al. (1985). Os dados de reflectância das amostras, na faixa de comprimento de onda de 1.100 a $2.500 \mathrm{~mm}$, foram armazenados por um espectrômetro (modelo NR5000: NIRS Systems, Inc., USA) acoplado a um microcomputador. Os valores de PB e DIVMO foram obtidos por equações de calibração desenvolvidas a partir de análises do capim realizadas por métodos convencionais (Euclides \& Medeiros, 2003).

Antes da amostragem anterior, foram coletadas aproximadamente 100 folhas diagnósticas (lâminas das duas folhas recém-expandidas do ápice para a base, com lígula visível) de cada parcela para análise de macronutrientes nas plantas, conforme Sarruge \& Haag (1974), com exceção do $\mathrm{N}$, que foi determinado pelo NIRS. Após a amostragem, foram realizadas as operações de corte, remoção da biomassa e adubação de reposição $\left(100 \mathrm{~kg} / \mathrm{ha}\right.$ de $\mathrm{K}_{2} \mathrm{O}$ com $\mathrm{KCl}$ ). Em agosto de 2004, foram retiradas amostras de solo nas 64 parcelas (0 a $20 \mathrm{~cm}$ ) para análise química, realizada conforme Embrapa (1997).

Os dados foram analisados por meio de análise de variância e regressão usando os procedimentos GLM e REG do programa SAS (1993). Foram ajustadas funções de resposta, pelas médias, do tipo: $\mathrm{Y}=\mathrm{bo}+\mathrm{b}_{1} \mathrm{~V}+\mathrm{b}_{2} \mathrm{~V}^{2}+\mathrm{b}_{3} \mathrm{P}+$ $\mathrm{b}_{4} \mathrm{P}^{2}+\mathrm{b}_{5} \mathrm{~N}+\mathrm{b}_{6} \mathrm{~N}^{2}+\mathrm{b}_{7} \mathrm{VP}+\mathrm{b}_{8} \mathrm{VN}+\mathrm{b}_{9} \mathrm{PN}$, adotando-se somente os coeficientes significativos pelo teste t.Nocaso da produção de massa seca verde (MSV) da forragem (folhas e colmo + bainhas verdes), as doses e combinações de nutrientes para máxima produção foram obtidas por cálculo diferencial $\partial \mathrm{Y} / \partial \mathrm{V}=0, \partial \mathrm{Y} / \partial \mathrm{P}=0$ e $\partial \mathrm{Y} / \partial \mathrm{N}=0$.
Para a máxima produção econômica, foram calculadas $\partial \mathrm{Y} / \partial \mathrm{P}=\mathrm{C}_{\mathrm{P}} / \mathrm{C}_{\mathrm{MSV}}$ e $\partial \mathrm{Y} / \partial \mathrm{N}=\mathrm{C}_{\mathrm{N}} / \mathrm{C}_{\mathrm{MSV}}$, em que $\mathrm{C}_{\mathrm{MSV}}$ éo preço de $1 \mathrm{~kg}$ de $\mathrm{MSV}$ da forragem e $\mathrm{C}_{\mathrm{P}}$ e $\mathrm{C}_{\mathrm{N}}$, o preço de $1 \mathrm{~kg}$ de $\mathrm{P}_{2} \mathrm{O}_{5}$ e $\mathrm{N}$, respectivamente. Essa análise não foi feita para a saturação por bases, uma vez que as quantidades de calcário utilizadas foram variáveis por parcela do ensaio. Assim, o nível de saturação por bases adotado foi o calculado para a máxima produção agronômica.

Para os cálculos de retorno econômico, foram considerados os seguintes preços: $\mathrm{MSV}$ : $\mathrm{R} \$ 0,10 / \mathrm{kg} ; \mathrm{P}_{2} \mathrm{O}_{5}$ : $\mathrm{R} \$ 2,00 / \mathrm{kg}$ dividido por 24 meses, tendo em vista sua ação residual, e multiplicado pelo total de meses da fase avaliada; $\mathrm{N}: \mathrm{R} \$ 2,00 / \mathrm{kg}$. O valor da forragem foi estimado em relação ao ganho de peso de bovinos em recria sob pastejo, conforme resultados da Embrapa (2001) em capim-massai e estimativas de Martha Jr. et al. (2004b). O preço do kg de peso vivo foi estimado em $\mathrm{R} \$ 2,10$.

\section{Resultados e Discussão}

Os dados de acúmulo de forragem apresentados nesse trabalho (Tabela 3) são de massa seca verde (MSV), os quais são mais correlacionados que a massa seca total com o consumo e ganho de peso dos animais nas pastagens (t'Mannetje, 1974). Na fase do estabelecimento da gramínea, as análises de variância e regressão indicaram resposta significativa linear e quadrática para $\mathrm{P}(\mathrm{P}<0,05)$. A maior resposta relativa na produção de MSV ocorreu até a dose de $80 \mathrm{~kg} / \mathrm{ha}$ de $\mathrm{P}_{2} \mathrm{O}_{5}$, correpondendo, na média, a 25,62 $\mathrm{kgMSV} / \mathrm{kgP}_{2} \mathrm{O}_{5}$ (Tabela 3).

A resposta positiva na produção das forrageiras com o uso de $\mathrm{P}$ em solo de Cerrado é esperada, especialmente quando os teores de $\mathrm{P}$ encontrados no solo são baixos. Essa ocorrência está de acordo com diversos trabalhos (Corrêa 
\& Haag, 1993; Santos et al., 2002). A menor resposta relativa $\left(\mathrm{kgMSV} / \mathrm{kgP}_{2} \mathrm{O}_{5}\right.$ ) ao aumento das doses igualmente foi verificada em vários trabalhos (Correa \& Haag, 1993; Fonseca et al., 1997). Correa et al. (1996), avaliando quatro cultivares de Panicum, inclusive o cultivar Massai, no estabelecimento, verificaram que a dose de $\mathrm{P}_{2} \mathrm{O}_{5}$ associada a $80 \%$ de produção máxima foi de $223 \mathrm{~kg} / \mathrm{ha}$, semelhante para os cultivares testados.

A função de resposta (Figura 2) e os dados médios de acúmulo de MSV nos níveis de $\mathrm{P}$ e saturação por bases (Tabela 4) permitem verificar que, além do P, a saturação por bases também contribuiu na produção do capimmassai. Os coeficientes linear e quadrático da saturação por bases não foram significativos no nível de $10 \%$ pelo teste $\mathrm{t}$, normalmente adotado para os coeficientes da equação de regressão, no entanto, situaram-se próximos $(0,10>\mathrm{P}<0,20)$. Houve aumento consistente do acúmulo de MSV com o aumento dos níveis da saturação por bases, especialmente nas duas menores doses de P (Tabela 4). Além disso, do ponto de vista agronômico, não parece razoável subestimar a oportunidade da prática da calagem com incorporação do calcário no solo durante o estabelecimento da pastagem em solo ácido.

A resposta ao fósforo ocorreu em todos os níveis de saturação por bases (Tabela 4). São freqüentes respostas positivas à aplicação de $\mathrm{P}$ pelas gramíneas forrageiras tropicais mesmo em níveis baixos de saturação por bases, tendo em vista a tolerância destas plantas à acidez (Luz et al., 2000).

O aumento do acúmulo de MSV em maior saturação por bases, ocorrido principalmente nas duas primeiras doses de $\mathrm{P}$, pode ter sido ocasionado pelo aumento de disponibilidade de $\mathrm{Ca}$ e $\mathrm{Mg}$ e, também, pela maior disponibilidade de $\mathrm{P}$ e $\mathrm{N}$ às plantas, uma vez que nas doses mais altas de $\mathrm{P}$ o efeito da calagem não é evidente. Nessas doses,
Tabela 3 - Acúmulo estimado de MSV total de dois cortes. Médias de duas repetições

\begin{tabular}{|c|c|c|c|c|}
\hline \multirow[t]{2}{*}{$\mathrm{P}_{2} \mathrm{O}_{5}$} & \multirow[t]{2}{*}{$\mathrm{V}$} & \multirow[t]{2}{*}{$\mathrm{N}$} & \multicolumn{2}{|c|}{$\begin{array}{l}\text { Acúmulo de forragem } \\
\text { de capim-massai }\end{array}$} \\
\hline & & & Massa seca verde & Média \\
\hline $\mathrm{kg} / \mathrm{ha}$ & $\%$ & $\mathrm{~kg} / \mathrm{ha}$ & $\mathrm{kg} / \mathrm{ha}$ & $\mathrm{kg} / \mathrm{ha}$ \\
\hline 0 & 20 & 0 & 4.203 & \\
\hline 0 & 20 & 300 & 5.898 & \\
\hline 0 & 40 & 100 & 5.041 & \\
\hline 0 & 40 & 200 & 7.398 & \\
\hline 0 & 60 & 100 & 7.391 & \\
\hline 0 & 60 & 200 & 5.709 & \\
\hline 0 & 80 & 0 & 7.221 & \\
\hline 0 & 80 & 300 & 4.670 & 5.941 \\
\hline 80 & 20 & 100 & 6.739 & \\
\hline 80 & 20 & 200 & 7.751 & \\
\hline 80 & 40 & 0 & 8.812 & \\
\hline 80 & 40 & 300 & 8.119 & \\
\hline 80 & 60 & 0 & 8.763 & \\
\hline 80 & 60 & 300 & 7.677 & \\
\hline 80 & 80 & 100 & 7.893 & \\
\hline 80 & 80 & 200 & 8.173 & 7991 \\
\hline 160 & 20 & 100 & 7.534 & \\
\hline 160 & 20 & 200 & 8.562 & \\
\hline 160 & 40 & 0 & 9.580 & \\
\hline 160 & 40 & 300 & 8.770 & \\
\hline 160 & 60 & 0 & 8.070 & \\
\hline 160 & 60 & 300 & 8.191 & \\
\hline 160 & 80 & 100 & 7.950 & \\
\hline 160 & 80 & 200 & 9.668 & 8.541 \\
\hline 240 & 20 & 0 & 8.035 & \\
\hline 240 & 20 & 300 & 9.980 & \\
\hline 240 & 40 & 100 & 9.909 & \\
\hline 240 & 40 & 200 & 9.412 & \\
\hline 240 & 60 & 100 & 7.875 & \\
\hline 240 & 60 & 200 & 9.373 & \\
\hline 240 & 80 & 0 & 8.794 & \\
\hline 240 & 80 & 300 & 10.126 & 9.188 \\
\hline $\begin{array}{l}\text { Média } \\
\text { Teste F }\end{array}$ & & & $\mathrm{P}^{* *}{ }_{\mathrm{L}}^{7.915} \mathrm{P}_{\mathrm{Q}}^{*}$ & 7.915 \\
\hline $\mathrm{CV}$ & & & 0,0959 & \\
\hline
\end{tabular}

Em teste $F$ são indicados somente os coeficientes significativos nos níveis de $5 \%\left(^{*}\right)$ e $1 \%\left(^{* *}\right)$ de probabilidade.

$P_{L}, P_{Q}=$ Componentes linear e quadrático para $P$.

Tabela 4 - Acúmulo médio de MSV de capim-massai para as doses de $\mathrm{P}_{2} \mathrm{O}_{5}$ e níveis de saturação por bases calculada (V Calc ), com valores de $\mathrm{P}$ e saturação por bases resultantes no solo (V solo)

\begin{tabular}{|c|c|c|c|c|c|c|}
\hline \multirow[t]{2}{*}{ Doses de $\mathrm{P}_{2} \mathrm{O}_{5}$} & \multirow[t]{2}{*}{ P no solo (Mehlich 1) } & \multicolumn{4}{|c|}{ Nível de saturação por bases Calc (V solo) } & \multirow[t]{2}{*}{ Média } \\
\hline & & $20(11)$ & $40(28)$ & $60(39)$ & $80(48)$ & \\
\hline $\mathrm{kg} / \mathrm{ha}$ & $\mathrm{mg} / \mathrm{dm}^{3}$ & $-2-$ & - - & - - & - & \\
\hline 0 & 2,26 & 5.051 & 6.219 & 6.550 & 5.946 & 5.941 \\
\hline 80 & 3,21 & 7.245 & 8.466 & 8.220 & 8.033 & 7.991 \\
\hline 160 & 4,32 & 8.048 & 9.175 & 8.131 & 8.809 & 8.541 \\
\hline 240 & 5,43 & 9.008 & 9.661 & 8.624 & 9.460 & 9.188 \\
\hline Média & 3,80 & 7.338 & 8.380 & 7.881 & 8.062 & 7.915 \\
\hline
\end{tabular}


provavelmente ocorreu maior efeito do $\mathrm{P}$ na nutrição da planta e, também, maior mineralização da matéria orgânica liberando nutrientes, especialmente $\mathrm{N}$.

A falta de resposta ao $\mathrm{N}$ pode ter sido causada pelo suprimento deste nutriente pelo solo por meio da mineralização da MO decorrente do preparo do solo, da calagem e adubação e/ou pela baixa eficiência da adubação nas condições climáticas no período experimental. Saraiva \& Carvalho (1991) também não verificaram resposta expressiva a doses de $\mathrm{N}$ de até $120 \mathrm{~kg} / \mathrm{ha}$ no estabelecimento de capim-elefante em Latossolo Vermelho-Amarelo muito argiloso, no entanto, notaram resposta à adubação fosfatada. Esses autores atribuíram a falta de resposta ao $\mathrm{N}$ à mineralização da $\mathrm{MO}$, decorrente da alteração na dinâmica do $\mathrm{N}$ propiciada pelo preparo do solo e pela adubação com outros nutrientes.

O baixo volume de chuvas durante o verão (Figura 1), que alcançou apenas $227 \mathrm{~mm}$ entre o plantio e o primeiro corte, certamente prejudicou a produção de biomassa e pode ter comprometido eventual efeito do $\mathrm{N}$ aplicado no acúmulo de MSV, uma vez que, nestas condições climáticas, a absorção e utilização do $\mathrm{N}$ pode ter sido afetada e a perda por volatilização pode ter sido elevada. No segundo perío do de crescimento, a resposta das gramíneas forrageiras à adubação foi negativamente influenciada por fatores ambientais como diminuição da temperatura e radiação solar, típicos do outono.

Entre os fatores testados, somente a saturação por bases calculada (calagem) foi significativa na alteração da saturação por bases efetivamente encontrada no solo por meio da análise química (Figura 3). A ausência de efeito do

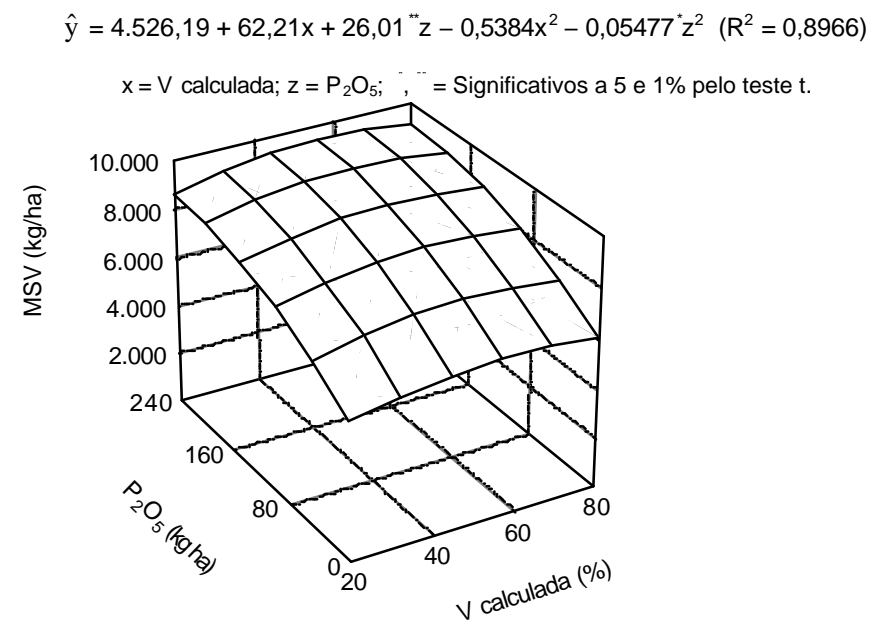

Figura 2 - Efeito das doses de $\mathrm{P}_{2} \mathrm{O}_{5}$ e da saturação por bases (V) calculada sobre o acúmulo de MSV de capimmassai.
$\mathrm{N}$, mesmo em suas maiores doses no estabelecimento, provavelmente resultou de seu baixo aproveitamento. A saturação por bases efetivamente encontrada no solo foi bastante inferior aos valores projetados. A dificuldade de atingir os valores alvos de saturação por bases no solo é freqüentemente relatada na literatura (Quaggio, 2000; Oliveira et al., 2003). Conforme Quaggio (2000), esse efeito ocorre quando são utilizados calcários tradicionais porque os carbonatos de $\mathrm{Ca}$ e $\mathrm{Mg}$ se tornam menos solúveis com o aumento do pH em sítios do solo em que se localiza o calcário aplicado, ocorrendo o efeito tamponamento; posteriormente, de forma gradativa, ocorre a reação destes carbonatos, em médio prazo (1 a 2 anos).

$\mathrm{Na}$ função de resposta para os valores de $\mathrm{P}$ extraídos do solo por Mehlich 1, houve efeito significativo apenas das doses de $\mathrm{P}_{2} \mathrm{O}_{5}$ aplicadas (Figura 4). O maior valor estimado foi de $5,40 \mathrm{mg} / \mathrm{dm}^{3}$ na dose máxima de $\mathrm{P}_{2} \mathrm{O}_{5}$ utilizada (240 kg/ha).

O valor estimado de saturação por bases no solo, no ponto de máximo acúmulo de MSV (Tabela 5) está de acordo com a recomendação da Embrapa (2001) para o estabelecimento de pastagem de capim-massai (40-45\%). Os valores de $\mathrm{P}$ no solo (Figura 4) nas doses de $\mathrm{P}_{2} \mathrm{O}_{5}$ que proporcionaram o ótimo desempenho econômico $\left(4,67 \mathrm{mg} / \mathrm{dm}^{3}\right)$ e agronômico $\left(5,36 \mathrm{mg} / \mathrm{dm}^{3}\right)$ foram menores que o recomendado (>6 mg/dm $\mathrm{de}^{3}$ P em Mehlich 1) para o estabelecimento dessa gramínea (Embrapa, 2001). A alta capacidade de fixação de P pelos solos argilosos do Cerrado (Novais \& Smith, 1999) provavelmente foi responsável por estes teores, que foram menores que o esperado para as doses de $\mathrm{P}_{2} \mathrm{O}_{5}$ utilizadas.

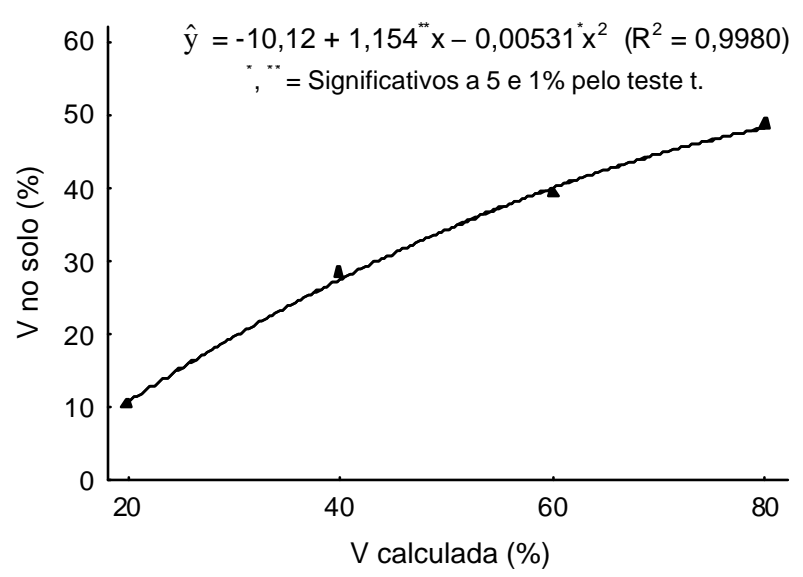

Figura 3 - Saturação por bases (V) no solo em relação à saturação por bases calculada. 
A avaliação econômica trata-se de um indicativo da melhor dose de $\mathrm{P}_{2} \mathrm{O}_{5}$, por isso, é denominada critério do capital ilimitado, que busca maximizar o lucro com a maximização do uso de insumos, pressupondo não existir restrições de capital e outros recursos e que não há riscos envolvidos (Alvarez V., 1985).

Em $20 \%$ de saturação por bases calculada, o pH $(4,38)$, o $\mathrm{Mg}\left(3,05 \mathrm{mmol}_{\mathrm{c}} / \mathrm{dm}^{3}\right)$ e o $\mathrm{Ca}\left(5,00 \mathrm{mmol} / \mathrm{dm}^{3}\right)$ estimados (Figura 5) mantiveram-se em níveis baixos (Macedo, 2005), enquanto os valores mais altos do $\mathrm{Al}\left(5,63 \mathrm{mmol}_{\mathrm{c}} / \mathrm{dm}^{3}\right)$ e sua saturação no solo $(37,5 \%)$, que igualmente ocorrem na saturação por bases calculada de $20 \%$ (Figura 6), encontram-se abaixo de diversos relatos de valores associados ao nível crítico (NC) de gramíneas tropicais (Cantarutti \& Novais, 2005) e dificilmente foram limitantes ao capim-massai.

Segundo Monteiro (2005), deve-se realizar a análise química foliar para avaliar o estado nutricional das planta em folhas com desenvolvimento em período favorável. Assim, os resultados da análise das folhas diagnósticas do primeiro corte foram utilizados para ajustar equações tendo como variáveis respostas os macronutrientes $\mathrm{N}, \mathrm{P}, \mathrm{Ca}$ e Mg. Esse autor relatou resultados de trabalhos em andamento

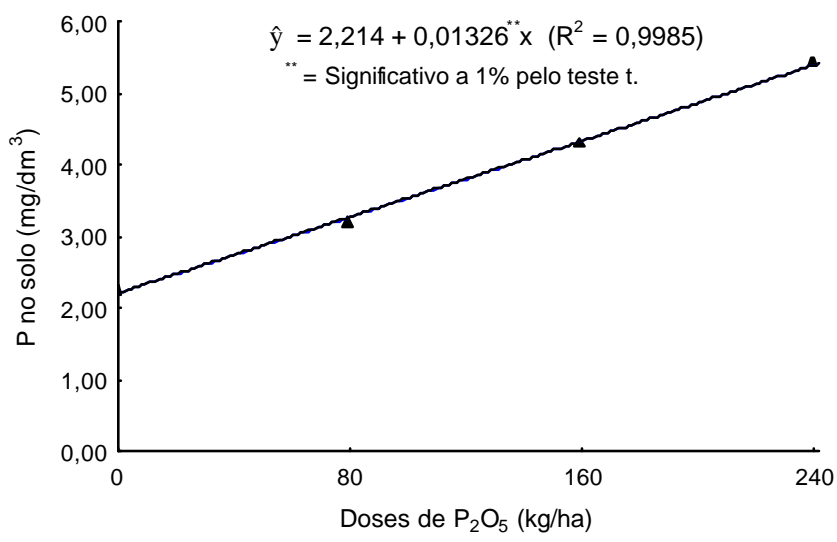

Figura 4 - Teor de P no solo extraído por Mehlich 1 (P no solo) nas doses de $\mathrm{P}$ aplicadas $\left(\mathrm{P}_{2} \mathrm{O}_{5}\right)$.

Tabela 5 - Saturação por bases calculada e no solo (V Calc e V solo) e doses de $\mathrm{P}\left(\mathrm{P}_{2} \mathrm{O}_{5}\right)$ aplicadas para máxima produtividade econômica e agronômica de MSV de capim-massai

\begin{tabular}{|c|c|c|c|}
\hline \multirow[t]{3}{*}{ Ponto de máximo } & \multirow{3}{*}{$\begin{array}{l}\text { Produtividade } \\
\qquad \text { - kg/ha- }\end{array}$} & \multicolumn{2}{|c|}{$\begin{array}{l}\text { Nível de saturação por } \\
\text { bases e doses de } \mathrm{P}_{2} \mathrm{O}_{5}\end{array}$} \\
\hline & & V Calc (V solo) & $\mathrm{P}_{2} \mathrm{O}_{5}$ \\
\hline & & $-\%$ & $-\mathrm{kg} / \mathrm{ha}-$ \\
\hline Máximo agronômico & 9.411 & $58(39)$ & 237 \\
\hline Máximo econômico & 9.260 & $58(39)$ & 185 \\
\hline
\end{tabular}

nos quais o NC de $\mathrm{P}$ para cultivares de $P$. maximum (Mombaça e Tanzânia) nas folhas diagnósticas variaram de 1,13 a $1,36 \mathrm{~g} / \mathrm{kg}$.

$\mathrm{O}$ valor máximo da concentração de $\mathrm{P}$ foliar no capimmassai $(1,23 \mathrm{~g} / \mathrm{kg})$ foi atingido no maior nível de saturação por bases calculada e na maior dose de $\mathrm{P}_{2} \mathrm{O}_{5}$ (Figura 7), pois extrapolou os intervalos testados. No menor nível de saturação por bases e na dose zero de $\mathrm{P}_{2} \mathrm{O}_{5}$, a concentração de $\mathrm{P}$ atingiu $0,88 \mathrm{~g} / \mathrm{kg}$, provavelmente uma condição de deficiência.

No caso do N, nas folhas diagnósticas, Manarim \& Monteiro (2002) verificaram NC de 16,0 a $16,5 \mathrm{~g} / \mathrm{kg}$ para o cultivar Mombaça e Abreu \& Monteiro (1999) verificaram NC de 21,4 g/kg para o cultivar Tanzânia. Os efeitos da adubação com $\mathrm{N}$ e da calagem foram significativos na concentração de N (Figura 8), mas a menor concentração do $\mathrm{N}$ foliar ainda permaneceu acima de $20,0 \mathrm{~g} / \mathrm{kg}$, que muito provavelmente não é limitante ao capim-massai.

As concentrações foliares de $\mathrm{Ca}$ e $\mathrm{Mg}$ (Figura 9) aparentemente estiveram acima dos níveis críticos $(\mathrm{Ca}: 4 \mathrm{~g} / \mathrm{kg}$; $\mathrm{Mg}: 1,5-4,2 \mathrm{~g} / \mathrm{kg}$ ) relatados por Monteiro (2005). Esses resultados contrastam com os valores estimados para o teor destes nutrientes no solo.

Um possível efeito foi o aumento das concentrações foliares de $\mathrm{Ca}$ e $\mathrm{Mg}$ com o avanço da idade dos tecidos, a época do ano e a diminuição do ritmo de crescimento das plantas, conforme verificado por Macedo \& Euclides (1997) e Santos Jr. et al. (2005).

Para a DIVMO na fração folha, os efeitos dos níveis de saturação por bases e das doses de $\mathrm{N}$ foram significativos na média dos dois cortes. O valor máximo estimado foi de $66 \%$ na máxima saturação por bases calculada e na maior dose de $\mathrm{N}$; nos níveis inferiores de correção e adubação, o valor estimado foi de $62 \%$ (Figura 10).

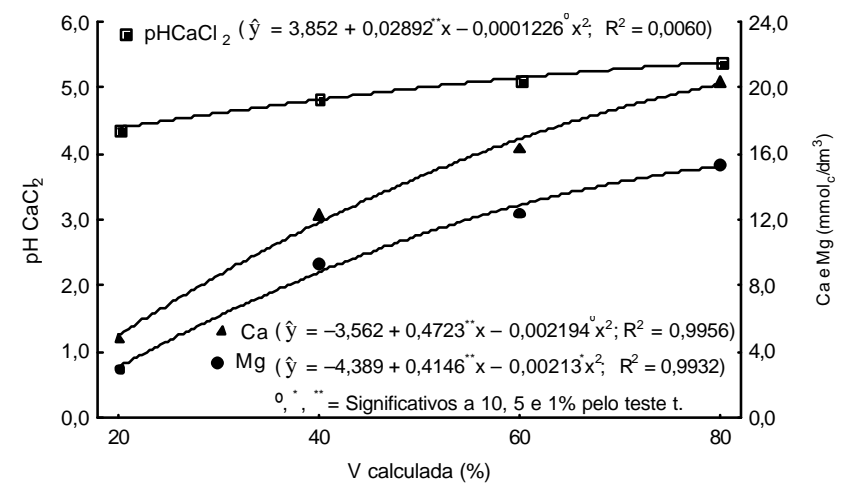

Figura 5 - Valores de $\mathrm{pH}$ em $\mathrm{CaCl}_{2}, \mathrm{Ca}$ e $\mathrm{Mg}$ no solo na saturação por bases calculada $(\mathrm{V})$. 
O teor de PB na fração folha estimado pela regressão (médias de dois cortes) variou de 13,72\% para as os valores máximos da saturação por bases calculada e dose de $\mathrm{N}$, até $11,94 \%$ para $20 \%$ de saturação por bases calculada e zero de N (Figura 11). Euclides \& Medeiros (2003) encontraram variações de 60,8 a 52,2\% para a DIVMO e 13,9 a 9,2\% para a PB em quatro épocas do ano, na média de quatro anos, em amostras simulando o pastejo de capim-massai. Correa et al. (2005), em ensaio de campo com capim-marandu, verificaram que o incremento das doses de $\mathrm{N}$ aumentou significativamente o teor de PB e a DIVMO em cortes realizados aproximadamente a cada 43 dias.

Os valores da DIVMO e PB foram obtidos na fração folha na fase de estabelecimento e em sistema de corte,

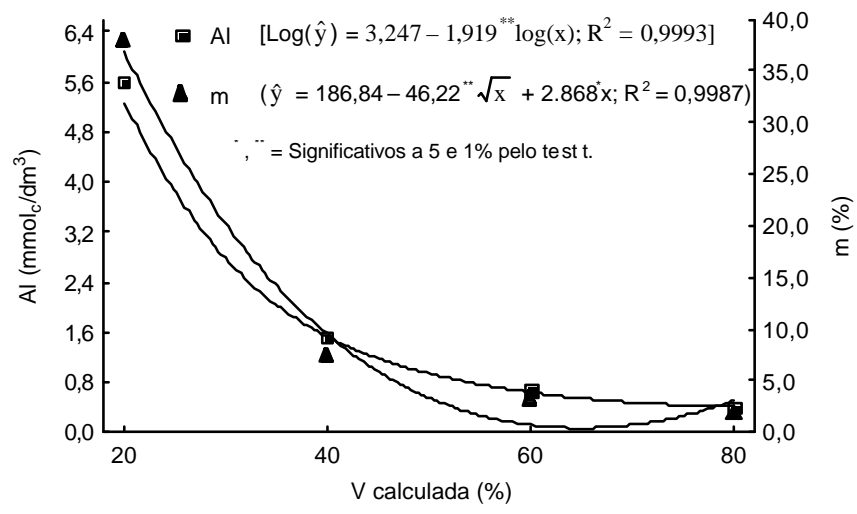

Figura 6 - Valores Al e da saturação por Al (m) no solo na saturação por bases calculada (V).

$\hat{y}=0,8488+0,00129^{* *} x+0,001943^{* *} z-0,000004^{* *} z^{2} \quad\left(R^{2}=0,8938\right)$

$\mathrm{x}=\mathrm{V}$ calculada $; \mathrm{z}=\mathrm{P}_{2} \mathrm{O}_{5} ; \quad=$ Significativo a $1 \%$ pelo teste $\mathrm{t}$.

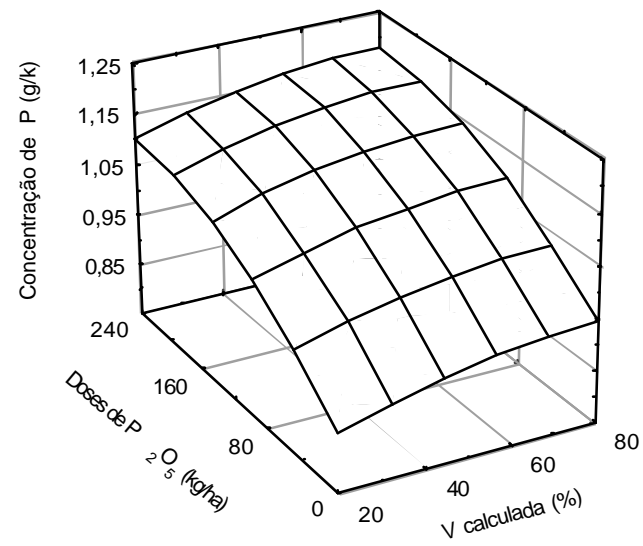

Figura 7 - Concentração de P nas folhas diagnósticas de capimmassai estabelecido em diferentes níveis de saturação por bases $(\mathrm{V})$ e doses de $\mathrm{P}\left(\mathrm{P}_{2} \mathrm{O}_{5}\right)$. condições que provavelmente justificam esses valores acima da média das gramíneas tropicais (Minson \& Wilson, 1980; Minson, 1990).

O valor nutritivo pode ser afetado negativamente por arranjos anatômicos verificados nas lâminas foliares do capim-massai, como a alta freqüência de estrutura girder $I$ e valores de bainha parenquimática dos feixes superiores aos do mesófilo (Lempp et al., 2004). Esses atributos podem afetar a facilidade e extensão da quebra do material, além do seu padrão no processo de consumo e digestão em ruminantes (Wilson, 1997). No entanto, a resistência de determinadas estruturas anatômicas à redução do tamanho das partículas pode não ser detectada pelas análises, pois podem ser destruídas na moagem da amostra

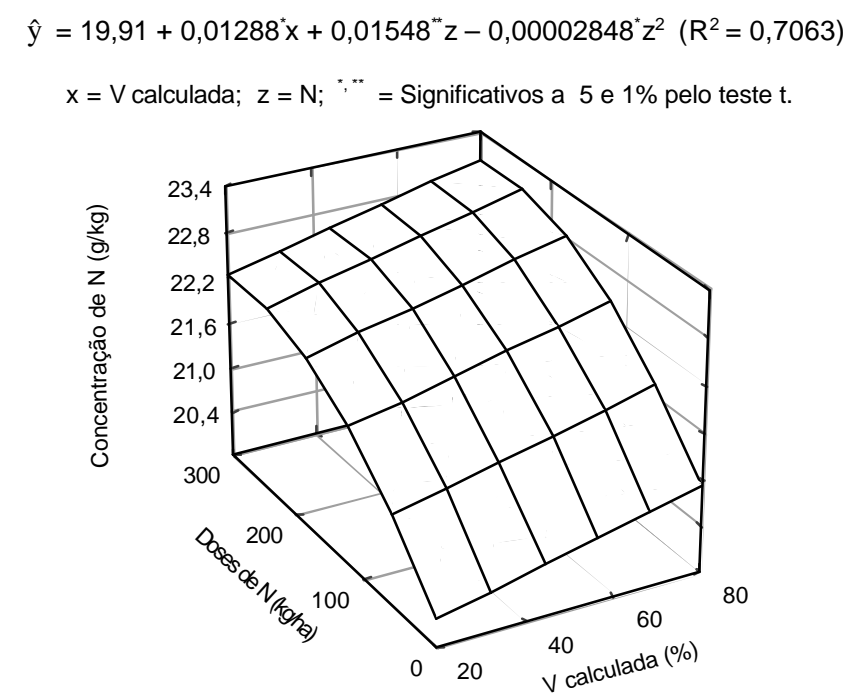

Figura 8 - Concentração de $\mathrm{N}$ nas folhas diagnósticas de capim-massai estabelecido em diferentes níveis de saturação por bases (V) calculada e doses de N.

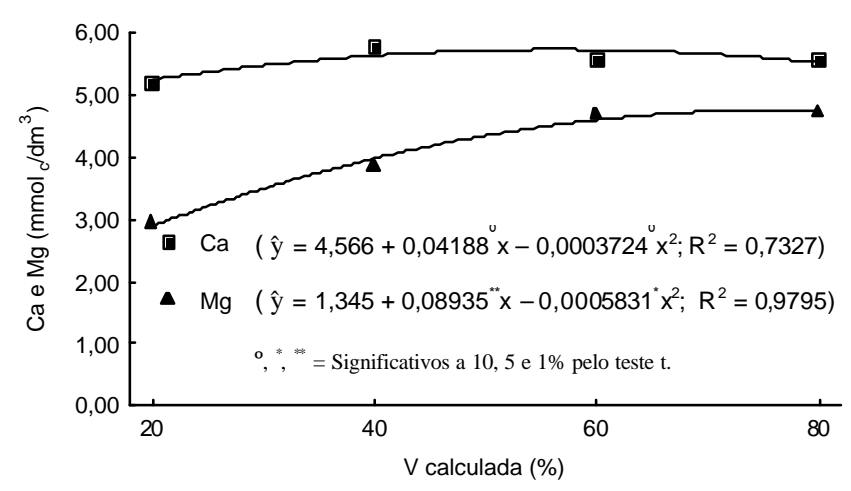

Figura 9 - Concentração de Ca e Mg nas folhas diagnósticas de capim-massai estabelecido em diferentes níveis de saturação por bases (V).

(c) 2008 Sociedade Brasileira de Zootecnia 
(Lempp et al., 2000). Portanto, os resultados das características químicas do $\mathrm{VN}$ verificados nesse trabalho exigem cautela.

$\mathrm{Na}$ média de todos os tratamentos e dos dois cortes realizados, a participação dos componentes morfológicos estimados foi: $\mathrm{MSV}=87,30 \%$ da massa seca total e massa seca de folhas $=77,50 \%$ da MSV. Deve-se ponderar que o intervalo de cortes neste trabalho foi prolongado, o que, por outro lado, permite verificar que esse capim é capaz de manter uma composição morfológica favorável por períodorelativamente longo, embora Martuscello et al. (2006) tenham verificado duração média de vida das folhas de aproximadamente

$$
\begin{gathered}
\hat{y}=60,35+0,06438^{\prime \prime \prime} x+0,01775^{\prime \prime} z-0,0002009^{\prime \prime} x z \quad\left(R^{2}=0,9206\right) \\
x=V \text { calculada } ; z=N ; \quad "=\text { Significativo a } 1 \% \text { pelo teste } t .
\end{gathered}
$$

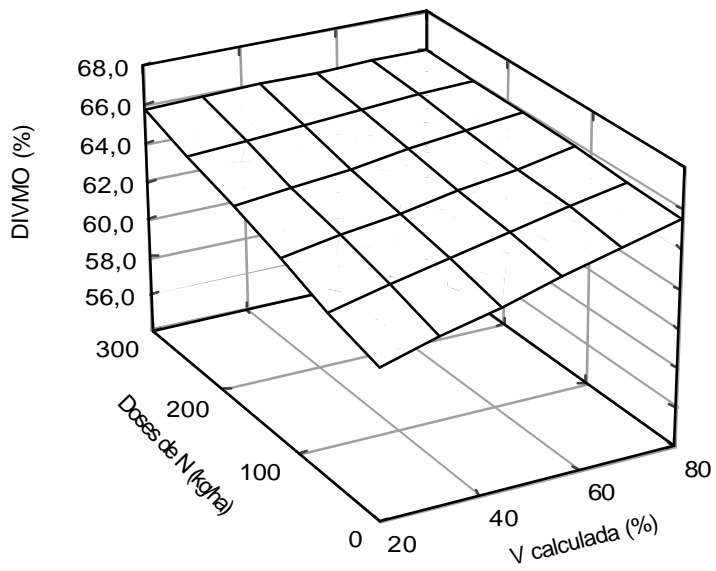

Figura 10 - DIVMO das folhas de capim-massai estabelecido em diferentes níveis de saturação por bases $(\mathrm{V})$ e doses de N.

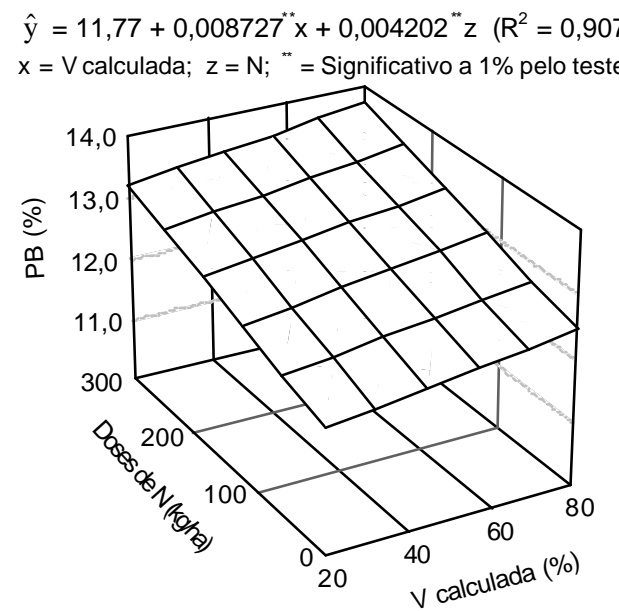

Figura 11 - Teor de PB nas folhas de capim-massai estabelecido em diferentes níveis de saturação por bases $(\mathrm{V})$ e doses de $\mathrm{N}$.
40 dias em condições favoráveis. Essa alta proporção de folhas na composição morfológica da biomassa é uma característica importante do capim-massai (Valentim et al., 2001).

\section{Conclusões}

$\mathrm{O}$ indicativo de dose econômica do fósforo $\left(\mathrm{P}_{2} \mathrm{O}_{5}\right)$ para o estabelecimento do capim-massai é de $185 \mathrm{~kg} / \mathrm{ha}$ em solo com $39 \%$ de saturação por bases, no qual ocorre aumento do acúmulo de massa seca verde até a dose de $237 \mathrm{~kg} / \mathrm{ha}$ de $\mathrm{P}_{2} \mathrm{O}_{5}$. A adubação nitrogenada no estabelecimento do capim-massai em preparo de solo convencional não é significativa para acúmulo de massa seca verde. A calagem e adubação com $\mathrm{P}$ melhoram significativamente a fertilidade do solo, enquanto a calagem e adubação com $\mathrm{P}$ e $\mathrm{N}$ aumentam significativamente as concentrações de N, P, Ca e Mg nas folhas diagnósticas; os efeitos mais importantes são os aumentos na concentração do $\mathrm{P}$ foliar e no teor de P no solo. A DIVMO e o teor de PB da fração folha respondem positivamente à adubação nitrogenada e ao aumento da saturação por bases e apresentam valores adequados na fase de estabelecimento dessa forrageira.

\section{Literatura Citada}

ABREU, J.B.R.; MONTEIRO, F.A. Produção e nutrição do capimmarandu em função da adubação nitrogenada e estádios de crescimento. Boletim de Indústria Animal, v.56, p.137-146, 1999.

ALVAREZ V., V.H. Avaliação da fertilidade do solo. Superfícies de resposta - modelos aproximativos para expressar a relação fator - resposta. Viçosa, MG: Universidade Federal de Viçosa, 1985. 75p.

ANDRADE, D.F.; NOLETO, A.Q. Exemplos de fatoriais fracionados $(1 / 2) 4^{3}$ e $(1 / 4) 4^{4}$ para o ajuste de modelos polinomiais quadráticos. Pesquisa Agropecuária Brasileira, v.21, n.6, p.677-680, 1986.

BRÂNCIO, P.A.; NASCIMENTO JR., D.; EUCLIDES, V.P.B. et al. Avaliação de três cultivares de Panicum maximum Jacq. sob pastejo: composição da dieta, consumo de matéria seca e ganho de peso animal. Revista Brasileira de Zootecnia, v.32, n.5, p.1037-1044, 2003.

CANTARUTTI, R.B.; NOVAIS, R.F. Quantificação da necessidade de uso de corretivo e fertilizante em pastagens. In: SIMPÓSIO SOBRE MANEJO DA PASTAGEM: Teoria e prática da produção animal em pastagens, 22., 2005, Piracicaba. Anais... Piracicaba: Fundação de Estudos Agrários Luiz de Queiroz, 2005. p.181-194.

CORREA, L.A.; HAAG, H.P. Níveis críticos de fósforo para o estabelecimento de gramíneas forrageiras em Latossolo vermelho amarelo, álico: II. Experimento de campo.Scientia Agrícola, v.50, n.1, p.109-116, 1993.

CORREA, L.A.; FREITAS, A.R.; EUCLIDES, V.P.B. Níveis críticos de fósforo para o estabelecimento de quatro cultivares de Panicum maximum em Latossolo Vermelho Amarelo, álico, In: REUNIÃO ANUAL DA SOCIEDADE BRASILEIRA DE ZOOTECNIA, 33., 1996, Fortaleza. Anais... Fortaleza: Sociedade Brasileira de Zootecnia, 1996. v.2, p.169.

CORREA, L.A.; PRIMAVESI, A.C.; PRIMAVESI, O. et al. Valor nutritivo de Brachiaria brizantha cv. Marandu em função de fontes e doses de nitrogênio. In: REUNIÃO ANUAL DA 
SOCIEDADE BRASILEIRA DE ZOOTECNIA, 42., 2005, Goiânia. Anais... Goiânia: Sociedade Brasileira de Zootecnia/ UFG Virtual, [2005]. (CD-ROM).

CRUZ, M.C.P.; FERREIRA, M.E.; LUCHETA, S. Efeito da calagem sobre a produção de matéria seca de três gramíneas forrageiras. Pesquisa Agropecuária Brasileira, v.29, n. 8, p.303-312, 1994.

EMPRESA BRASILEIRA DE PESQUISA E AGROPECUÁRIA EMBRAPA. Manual de métodos de análise de solo. 2.ed. Rio de Janeiro: Centro Nacional de Pesquisa de Solos, 1997, 212p. (Documentos, 1).

EMPRESA BRASILEIRA DE PESQUISA E AGROPECUÁRIA EMBRAPA. Capim-massai (Panicum maximum cv Massai): alternativa para diversificação de pastagens. Campo Grande: Embrapa Gado de Corte, 2001. 8p. (Comunicado Técnico, 65).

EUCLIDES, V.P.B.; MEDEIROS, S.R. Valor nutritivo das principais gramíneas cultivadas no Brasil. Campo Grande: Embrapa Gado de Corte, 2003, 43p. (Documentos, 139).

FONSECA, D.M.; GOMIDE, J.A.; ALVAREZ V., V.H. et al. Fatores que influenciam os níveis críticos de fósforo para o estabelecimento de gramíneas forrageiras. II. Em campo. Revista Brasileira de Ciência do Solo, v.21, p.35-40, 1997.

LEMPP, B.; EUCLIDES, V.P.B.; MORAIS, M.G. et al. Avaliação do resíduo da digestão de três cultivares de Panicum maximum. In: REUNIÃO ANUAL DA SOCIEDADE BRASILEIRA DE ZOOTECNIA, 37., 2000, Viçosa, MG. Anais... São Paulo: Sociedade Brasileira de Zootecnia/Gmosis, [2000]. (CD-ROM).

LEMPP, B.; KICHEL, A.G.; GOMES, R. et al. Proporção e arranjo de tecidos em lâminas foliares dePanicum maximum cv Massai. In: REUNIÃO ANUAL DA SOCIEDADE BRASILEIRA DE ZOOTECNIA, 41., 2004, Campo Grande. Anais... Campo Grande: Sociedade Brasileira de Zootecnia/Embrapa Gado de Corte, [2004]. (CD-ROM).

LUZ, P.H.C.; HERLING, V.R.; BRAGA, G.J. et al. Efeitos de tipos, doses e incorporação de calcário sobre características agronômicas e fisiologias do capim-tobiatã (Panicum maximum Jacq.). Revista Brasileira de Zootecnia, v.29, n.4, p.964970, 2000

MACEDO, M.C.M. Pastagens no ecossistema Cerrados: evolução das pesquisas para o desenvolvimento sustentável. In: REUNIÃO ANUAL DA SOCIEDADE BRASILEIRA DE ZOOTECNIA, 42., 2005, Goiânia. Anais... Goiânia: Sociedade Brasileira de Zootecnia, 2005. p.56-84.

MACEDO, M.C.M.; EUCLIDES, V.P.B. Changes in soil fertility and plant nutrient contents in degraded tropical pasture after renovation. In: INTERNATIONAL GRASSLAND CONGRESS, 18., 1997, Winnipeg, Saskatoon. Proceedings... Winnipeg: Soil, Fertility and Plant Nutrition/ICG, 1997. v.1, p.115-116.

MANARIM, C.A.; MONTEIRO, F.A. Nitrogênio na produção e diagnose foliar do capim-Mombaça. Boletim da Indústria Animal, v.59, p.115-123, 2002.

MARTEN, G.C.; SHENK, J.S.; BARTON II, F.E. Near infrared reflectance spectroscopy (NIRS), analysis of forage quality. Washington: USDA, ARS, 1985. 110p. (Agriculture Handbook, 643).

MARTHA JR., G.B.; CORSI, M.; TRIVELIN, P.C.O. Nitrogen recovery and loss in a fertilized elephantgrass pasture. Grass and Forage Science, v.59, p.80-90, 2004a.

MARTHA JR., G.B.; VILELA, L.; BARIONI, L.G. et al. Manejo e adubação nitrogenada em pastagens In: SIMPÓSIO SOBRE MANEJO DA PASTAGEM: Fertilidade do solo para pastagens produtivas, 21., 2004, Piracicaba. Anais... Piracicaba: Fundação de Estudos Agrários Luiz de Queiroz, 2004b. p.155216.

MARTUSCELLO, J.A.; FONSECA, D.M.; NASCIMENTO JR., D. et al. Características morfogênicas e estruturais de capim-massai submetido a adubação nitrogenada. Revista Brasileira de Zootecnia, v.35, n.3, p.665-678, 2006.
MINSON, D.J. Forage in ruminant nutrition. San Diego: Academic Press, 1990. 483p.

MINSON, D.J.; WILSON, J.R. Comparative digestibility of tropical and temperate forage - a contrast between grasses and legumes. The Journal of the Australian Institute of Agricultural Science, v.46, n.4, p.247-249, 1980.

MONTEIRO, F.A. Amostragem de solo e de planta para fins de análises químicas: método de interpretação de resultados. In: SIMPÓSIO SOBRE MANEJO DE PASTAGENS: Teoria e prática da produção animal em pastagens, 22., 2005, Piracicaba. Anais... Piracicaba: Fundação de Estudos Agrários Luiz de Queiroz, 2005.p.151-180.

NORONHA, J.F. Teoria da produção aplicada à análise econômica de experimentos. In: CONTINI, E.; ARAÚJO, J.D.; OLIVEIRA, A.J. et al. (Eds.). Planejamento da propriedade agrícola. Modelos de decisão. Brasília: Embrapa DDT, 1984. p.2365

NOVAIS, R.F.; SMYTH, T.J. Fósforo em solo e planta em condições tropicais. 1.ed. Viçosa, MG: Universidade Federal de Viçosa, 1999. 299p.

OLIVEIRA, P.P.A.; BOARETTO, A.E.; TRIVELIN, P.C.O. et al. Liming and fertilization to restore degraded Brachiaria decumbens pastures grown on an entisol. Scientia Agrícola, v.60, n.1, p.125-131, 2003.

PAULINO, V.T.; COSTA, N.L.; RODRIGUES, A.N.A. et al. Resposta de Panicum maximum cv Massai à níveis de calagem. In: REUNIÃO ANUAL DA SOCIEDADE BRASILEIRA DE ZOOTECNIA, 43., 2006, João Pessoa.. Anais... João Pessoa: Sociedade Brasileira de Zootecnia, 2006. (CD-ROM).

PRIMAVESI, A.C.; PRIMAVESI, O.; GODOY, R. Resposta da aveia branca à adubação em Latossolo Vermelho-amarelo em dois sistemas de plantio. Revista Brasileira de Zootecnia, v.33, n.1, p.79-86, 2004.

QUAGGIO, J.A. Acidez e calagem em solos tropicais. Campinas: IAC, 2000. $111 \mathrm{p}$.

SANTOS, H.Q.; FONSECA, D.M.; CANTARUTTI, R.B. et al. Níveis críticos de fósforo no solo e na planta para gramíneas forrageiras tropicais, em diferentes idades. Revista Brasileira de Ciência do Solo, v.26, p.173-182, 2002.

SANTOS JR., J.D.; MACEDO, M.C.M.; MONTEIRO, F. et al. Concentração de macronutrientes em folhas diagnósticas de capim-tanzânia na rebrotação de verão e outono. In: REUNIÃO ANUAL DA SOCIEDADE BRASILEIRA DE ZOOTECNIA, 42. 2005, Goiânia. Anais...Goiânia: Sociedade Brasileira de Zootecnia/UFG Virtual, [2005]. (CD-ROM).

SARAIVA, O.F.; CARVALHO, M.M. Adubação nitrogenada e fosfatada para o estabelecimento de capim-elefante em Latossolo Vermelho-amarelo textura argilosa. Revista Brasileira de Ciência do Solo, v.15, n.2, p.201-205, 1991.

SARRUGE, J.R.; HAAG, H.P. Análises químicas em plantas. Piracicaba: Escola Superior de Agricultura Luiz de Queiroz, 1974. 56p.

STATISTICAL ANALYSIS SYSTEM - SAS. SAS/STAT - User's guide. Version 6, 4.ed. Cary: 1989. v.2, 846p.

t'MANNETJE, L. Relations between pastures attributes and live weight gains on a subtropical pasture. In: INTERNATIONAL GRASSLAND CONGRESS, 12., 1974, Moscow.Proceedings... Moscow: s.ed., 1974. p.299-304.

VALENTIM, J.F.; CARNEIRO, J.C.; MOREIRA, P. et al. Capimmassai (Panicum maximum Jacq): nova forrageira para diversificação das pastagens do Acre. Rio Branco: Embrapa Acre, 2001. 16p. (Circular Técnica, 41).

Van RAIJ, B. Fertilidade do solo e adubação. São Paulo; Piracicaba: Ceres, Potafós, 1991. 343p.

WILSON, J.R. Structural and anatomical traits of forages influencing their nutritive value for ruminants. In: SIMPÓSIO INTERNACIONAL SOBRE PRODUÇÃO ANIMAL EM PASTEJO, 1997, Viçosa, MG. Anais... Viçosa, MG: Universidade Federal de Viçosa, 1997. p.411-429. 